\title{
THE INVESTIGATION AND EVALUATION MULTISERVICE NETWORK NGN/IMS FOR MULTIMEDIA TRAFFIC
}

\author{
Bayram G. Ibrahimov \\ Azerbaijan Technical University,Baku, Azerbaijan, i.bayram@mail.ru \\ Arif H. Hasanov \\ Military Academy of the Republic of Azerbaijan, Baku, Azerbaijan
}

DOI: $10.36724 / 2664-066 X-2020-6-2-12-15$

\begin{abstract}
The functional architecture of the subsystem picture messaging-IMS (Internet Protocol Multimedia Subsystem) for multimedia session management for multiservice NGN/IMS networks that determine the interaction systems and NGN protocols are investigated. The basic element of the IMS core network architecture are session control function CSCF (Call/Session Control Function) which is implemented on the SIP-server (Session Initiation Protocol) using the protocol.

A block diagram of the functioning of the traffic service model of multimedia services in the NGN/IMS network when establishing sessions that use the network elements of the user data server HSS (Home Subscriber Server), CSCF control system core, signaling and media gateways (SGW \& MGW, Signaling Gateway \& Media Gateway). The characteristics of the effectiveness of the IMS and found that in the NGN / IMS multimedia services network in real time significantly alter the traffic characteristics as a service, and efficiency, which requires new models and approaches to assess the main indicators of quality of the service Triple Play.

Based on the analysis of the quality NGN/IMS networks in the provision multimedia services, the mathematical model for evaluating the quality of services. This model takes into account the properties of self-similar random process with
\end{abstract}

Hurst index and describes the quality of functioning of NGN/IMS architectural concept. Based on the model studied when performing voice services with the ability to activate multimedia applications, video telephony, multimedia traffic, Triple Play services.

According to the study of mathematical models of NGN / IMS networks using SIP-servers analytical expressions to assess the indicators of a common set of operating characteristics of IP-based networks, which take into account the recommendations of ITU-T Y.1540 and Y.2000 and determined the average waiting time in the queue IMS system in the provision of multimedia services with the required parameters, providing a guaranteed quality of service QoS (Quality of Service). According to the latest recommendations of the ITU-T and based on SMO with queues can be defined by five network characteristics that are indicative of the effectiveness of multi-service NGN/IMS networks and an important indicator of QoS.

KEYWORDS: multimedia traffic, SIP protocol, IMS subsystem, HSS server, quality of service, Diameter protocol, SIP-server.

Information about authors

Ibrahimov Bayram Ganimat oglu, professor of Azerbaijan Technical University, Bak, Azerbaijan

Hasanov Arif Hasan oglu, an associate of the Military Academy of the Republic of Azerbaijan, Baku, Azerbaijan 


\section{Introduction}

At present, in the field of telecommunications, as the number of multimedia services and the volume of useful and service traffic increases, it is necessary to create effective multi-service communication networks of the next generation based on the multimedia IMS platform, which provide the necessary quality of services provided to subscribers.

Studies have shown $[1,2]$ that one of the most important tasks of multiservice NGN / IMS networks using SIP servers is to support the quality of service of multimedia traffic, primarily to minimize the time characteristics of service access delays. It should be noted that so far this issue has not been studied well enough and remains poorly studied [3,4].

The efficiency of the IMS multimedia communication subsystem largely depends on the capacity of NGN / IMS networks using SIP servers and network equipment of the CSCF session management system. To guarantee the service of such loads, maximum NGN / IMS network bandwidth, minimum average delays in the IMS system, increased efficient use of SIP server resources, etc. are required.

In view of the above, an important question arises - the assessment of the quality of service of the multimedia traffic of the IMS multimedia communication, which depends both on the service access algorithm and on the efficiency of the NGN / IMS multi-service networks using the HSS home subscriber server.

In this work, a study and evaluation of the effectiveness of multiservice NGN / IMS networks in the transmission of multimedia traffic is carried out.

\section{General problem statement and scheme of functioning the investigated model of the NGN / IMS network}

To accurately describe the multimedia traffic passing between the links of the NGN / IMS network and its network elements of the CSCF session control system, an analysis of the statistical characteristics of the traffic and the choice of an adequate MM of a self-similar random process is required. Analysis of the IMS concept $[1,5,6]$ showed that the further expansion of the range of multimedia services demanded by users has led to the emergence of a Triple Play Service package, which is a triple package that includes voice services, Internet access and viewing of television programs that require multi-rate service systems and a wide range of transmission rates - from 2 to $2500 \mathrm{Mbit} / \mathrm{s}$.

As part of the study, the structural and functional diagram of the NGN / IMS network model, built on the basis of the systemic and technical analysis of multimedia services, is shown in Fig. 1 and consists of an access buffer storage (BN), a switching node using IP / MPLS, and an IMS subsystem.

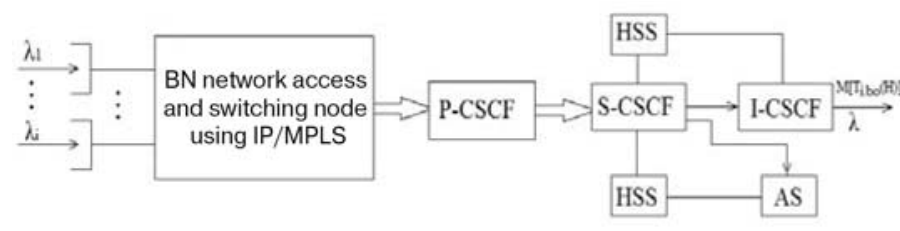

Figure 1. Structural and functional diagram of NGN / IMS network investigated model
The IMS multimedia communication subsystem as well as the Softswitch architecture, which is a set of functions connected by standard interfaces. The IMS system consists of Proxy, Interrogating and Serving CSCFs, which use SIP and Diameter IMS session establishment protocols.

In the scheme, the HSS is a user data base and provides access to personal data of subscribers associated with multimedia services. The AS communicate with the S-CSCF via SIP and maintain SIP sessions.

On the basis of studies $[3,5]$, it has been established that the transmitted traffic systems and protocols of NGN / IMS networks have a special structure. It was revealed in [2] that multimedia NGN / IMS traffic has self-similarity with the Hurst coefficient, $\mathrm{H}$, lying in the range of $0.5 \leq \mathrm{H}<1$ and is equal to $H=1-0,5 \beta, 0<\beta<1$ [3].

In order to solve the problem under consideration and taking into account the importance of the interaction of the system and the NGN protocols, a mathematical model (MM) of NGN / IMS networks is proposed, taking into account the properties of selfsimilarity of traffic when providing multimedia services.

\section{Description of the mathematical model and analysis of indicators of NGN / IMS networks}

Recently, the analyzes carried out show $[3,4,6]$ that the nature of the transmitted service and payload traffic in multiservice NGN / IMS networks differs from the Poisson one, and the service process from the exponential distribution law. Then it is necessary to turn to the results of the theory of diffusion approximation, which provides the developer with approximate, but very simple from the computational point of view, formulas [4].

Considering the last assumption and algorithms of the functional architecture of the multimedia messaging subsystem, the considered NGN / IMS networks, in the general case, is a queuing system (QS) of type $f B G I / G / N_{S} / N_{б н}$ with some assumptions at increased load $\rho_{i} \leq 1, i=\overline{1, n}$.

Suppose that the arrival of traffic flows for service is nonPoissonian with intensity $\lambda_{\mathrm{i}}, i=\overline{1, n}$, the distribution of the service duration is arbitrary, serving NS SIP servers have a common buffer storage (BN) with an unlimited capacity $N_{\text {бн }} \leq \infty$ (where $n$ - total number of traffic flow types).

Considered $\mathrm{MM}$ in the form of a general QS $f B G I / G / N_{S} / N_{б н}$ with queues, takes into account the performance indicators of NGN / IMS networks, the properties of selfsimilarity of multimedia traffic and the features of approximate methods of diffusion approximation.

Based on diffusion approximation methods and selfsimilarity properties of transmitted traffic in a given QS, the load of the multimedia message transmission subsystem is determined as follows:

$$
\rho_{i}\left(H, \lambda_{i}\right)=\left[B_{i}^{(1)} \cdot f(H) \cdot \lambda_{i} /\left(V_{i} \cdot N_{S}\right)\right] \leq 1, i=\overline{1, n}
$$

where $f(H)$ - function that takes into account the property of self-similarity of incoming traffic packets of the protocol of NGN / IMS networks; $B_{i}^{(1)}(t)$ - the average value of the duration 
of service of streams of packets of $i$-th traffic; $V_{i}$ - packet stream rate of $i$-th traffic.

For the purpose of convenient calculation, expression (1) can be presented in the following edition, introducing the load intensity of the multimedia messaging subsystem for the packet stream $i$-th traffic

$$
\rho(H)=\sum_{i=1}^{n} \rho_{i}\left(H, \lambda_{i}\right) \leq 1, \quad i=\overline{1, n}
$$

It should be noted that the idea and features of the diffusion approximation method described in $[4,5]$ is that the queue length distribution $P(n \geq 0)$ in system $f B G I / G / N_{S} / N_{б н}$ with queues at critical load equal to $\rho(H)$, is approximated by the following distribution:

$$
P(n \geq 0)=\left\{\begin{array}{lr}
1-\rho(H), & \text { nрu } n=0 \\
\rho(H)\left[1-\rho\left(C_{A}, C_{B}\right)\right] \cdot\left[\rho\left(C_{A}, C_{B}\right)^{n-1},\right. & \text { при } n \geq 0
\end{array},\right.
$$

In (3) $\rho\left(C_{A}, C_{B}\right)$ is a load of the CMO type $f B G I / G / N_{S} / N_{б н}$, taking into account the quadratic coefficients of variation of the distribution $C_{A}^{2}$ intervals between incoming multimedia messages and the distribution of lengths of multimedia messages $C_{B}^{2}$ and is expressed as follows:

$$
\rho\left(C_{A}, C_{B}\right)=\exp \left[-\frac{2[1-\rho(H)]}{\rho(H) \cdot C_{A}^{2}+C_{B}^{2}} \leq 1\right.
$$

Therefore, for the Poisson incoming stream of traffic packets $C_{A}^{2}=1$, and for the geometric distribution of message lengths is

$$
C_{B}^{2}=C_{B}^{2}\left(L_{c 3 . n}\right)=\left(\sigma_{L_{\text {сзол }}} / L_{c 3 . n}\right)^{2}=p_{c}
$$

where $p_{c}-$ the likelihood that the average value $L_{c 3 . n}$ not equal to the packet data unit for the geometric distribution of the length of multimedia messages; $\sigma_{L_{c, n}, n}^{2}$ - variance for the geometric distribution of the length of a multimedia message.

\section{Estimation of the transmission time of multimedia traffics}

In this IMS system, we assume that all network elements are loaded identically and the load factor of one SIP server, taking into account the self-similarity property of the incoming $i$-th traffic packet $\rho_{i}(H)$ in a multichannel system is determined by the following expression:

$$
\rho_{i}(H)=\left[\frac{L_{i . c 3 . n}}{V_{i} \cdot N_{S}} \cdot \mu_{i} \cdot \lambda_{i} \cdot B_{i}^{(1)}\right] \leq 1, i=\overline{1, n},
$$

where $L_{i . c 3 . n}$ - average value of the length of the transmitted multimedia message of the $i$-th traffic.

Taking into account (2),.., (7) and the results obtained using the diffusion approximation method, it is possible to determine the probabilistic-temporal characteristics of NGN / IMS networks [4, 5].

Based on the proposed $\mathrm{MM}$ in the form of a general QS $f B G I / G / N_{S} / N_{б н}$ with queues, the average queue length is expressed as follows:

$$
\begin{gathered}
E\left[L_{i . \partial o}, H\right]=0,5 G\left(\rho, N_{S}\right) \cdot \frac{C_{A}^{2}+C_{B}^{2}}{1-\rho_{i}(H)} . \\
{\left[B_{i}^{(1)} \cdot f(H) \cdot \lambda_{i} /\left(V_{i} \cdot N_{S}\right)\right], \quad i=\overline{1, n}}
\end{gathered}
$$

где $G\left(\rho, N_{S}\right)$ - является множителем и определяет вероятность того, что потоки пакетов трафика придя в подсистему мультимедийной связи IMS, застанет все SIP-серверов занятыми и встанет в очередь и определяется как $C$-формула Эрланга [4].

С учетом (8) и приближенной формулы Крамера и Лангенбах-Бельца [5], среднее значение время ожидания $i$-го потока пакета трафика в очереди определяется следующим выражением:

$$
E\left[T_{i, B o}(H)\right]=\frac{t_{S}}{N_{S}\left[1-\rho_{i}(H)\right]} \cdot G(\rho, H) \cdot \frac{C_{A}^{2}+C_{B}^{2}}{2}, i=\overline{1, n}
$$

where $t_{S}$ - average service time of NGN / IMS traffic packet flows.

Figure 2 shows the dependence of the average waiting time in the queue in the NGN / IMS network on the number of SIP servers and the transmission rate of multimedia traffic.

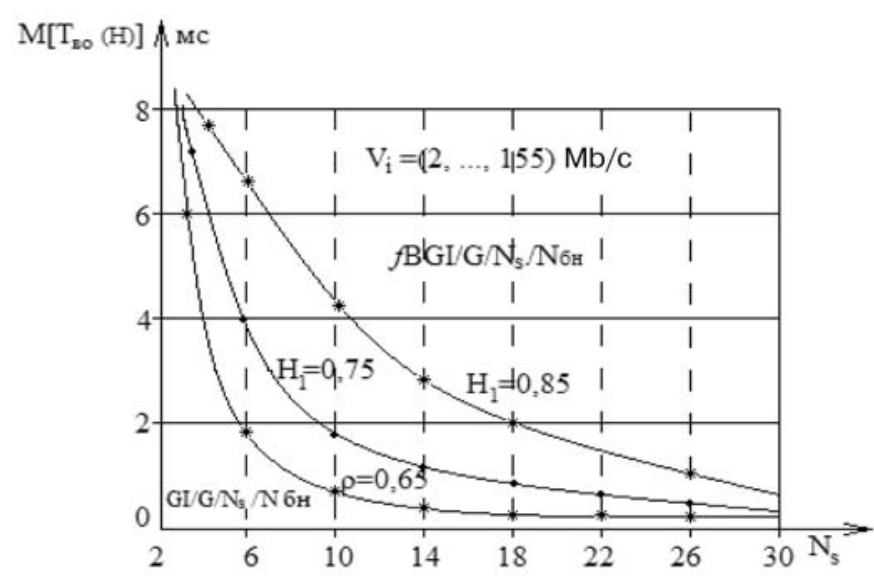

Figure 2. Dependence of the average waiting time in the queue in the NGN / IMS network on the number of SIP servers

Graphical pendency family analysis $E\left[T_{i .6 o}(H)\right]=F\left(\rho_{i}, N_{S}, V_{i . k}, H\right)$ shows that an increase in the limited number of SIP servers in the NGN / IMS network using HSS home subscriber servers $N_{S} \geq 20, \ldots, 25$, that meet the IMS fault tolerance requirements, help to minimize the average waiting time in the queue at a given speed $V_{i} \geq(2, \ldots, 155) \mathrm{Mbps}$ and Hurst coefficient $H_{i}=0,75, \ldots, 085$. 
Thus, based on MM, the obtained expressions (7), ..., (9) are one of the main characteristics of the NGN / IMS network efficiency and QoS indicators, which are based on the IMS architecture.

\section{Conclusions}

The conducted research has shown that assessments and analysis of the indicators of the IMS multimedia communication platform, both the average queue length of the queue system and the average delay time of traffic transmission in the load function, are very important when studying the effectiveness of NGN / IMS multiservice networks, taking into account the properties of self-similarity of service and useful traffic.

As a result of the study, an MM was proposed in the form of a general QS $f B G I / G / N / N_{\bar{~} H}$ with queues and analytical expressions were obtained that allow one to evaluate the performance indicators of NGN / IMS, taking into account the properties of self-similarity of multimedia traffic, ensuring guaranteed quality of QoS services, regulated by the ITU-T recommendations.

\section{References}

1. Fillimonov A.Yu. (2007). Construction of multi-service Ethernet networks. St. Petersburg: BVH - St. Petersburg, 592 p.

2. Ibrahimov B.G., Hasanov A.G., Ibrahimov R.F. (2016). Analysis of performance characteristics of multiservice communication networksbased on the IMS subsystem. Moscow: MTUCI, pp. 36-37. (in Russian)

3. Ibrahimov B.G., Hasanov A.G. (2016). Investigation of the quality of the functioning of NGN / IMS networks during the establishment of a multimedia session. Moscow: PFUR, 2016, pp. 21-24.

4. Basharin G.P.., Bocharov P.P., Kogan Ya.A. (1989). Analysis of queues in computer networks. Theory and methods of calculation. Moscow: Nauka. 336 p.

5. Gaidamaka Yu.V., Zaripova E.R., Vikhrova O.G. (2014). Approximate method of session initiation delay performance evaluation in IP multimedia subsystem. T-Comm, no.8, pp. 19-23.

6. Bosse J.G. Devetak F.U. (2007). Signaling in Telecommunications Networks. 2nd Ed. New York: Wiley. 830 p. 